\title{
Preface to the Proceedings of the First International Workshop on Self-Learning in Intelligent Environments
}

\author{
Antonio CORONATO ${ }^{\mathrm{a}, 1}$, Giovanna DI MARZO SERUGENDO ${ }^{\mathrm{b}}$ \\ ${ }^{a}$ Istituto di Calcolo e Reti ad Alte Prestazioni, CNR-ICAR, Italy \\ ${ }^{\mathrm{b}}$ University of Geneva, Switzerlanz.
}

Self-learning systems are artificial agents able to acquire and renew knowledge over the time by themselves, without any hard coding. These are adaptive systems whose functions improve by a learning process based -typically- on the method of trial and error. A self-learning system interacts with its users or surrounding environment initially by attempts and observes the changes produced by its actions.

This workshop focuses on the design, implementation and exploitation of selflearning features -either within an Intelligent Environment [2] as a whole, or within some of its components- by means of leading technologies $([8,6,7,5])$ and even in critical environments (e.g. healthcare $[3,1,4])$. The workshop will represent an opportunity for both the academia and industry to debate the state-of-the-art, challenges and open issues.

This first edition of the workshop has accepted for publication and presentation seven papers.

Aslan et al. have proposed an algorithm for learning to move the desired object by humanoid robots. In this algorithm, the semantic segmentation algorithm and Deep Reinforcement Learning (DRL) algorithms are combined. The semantic segmentation algorithm is used to detect and recognize the object be moved. DRL algorithms are used at the walking and grasping steps.

Donnici et al. have presented an intelligent system for supporting patients during their home medical treatment. The system can assist impaired patients in taking medicines in accordance with their treatment plans. The demonstration of the system via mobile app shows promising results and can improve the quality of healthcare at home.

Hayat et al. have proposed a framework that self-learns and automatically classifies any given news headline into its corresponding news category using artificial intelligence methods i.e. text mining and machine learning algorithms.

Ribino and Bonomolo have reported an approach based on reinforcement learning to support the rearrangement of indoor spaces by maximizing the indoor environmental quality index in terms of thermal, acoustic and visual comfort in the new furniture layout scheme.

Shah and Coronato have exploited an IRL method named Max-Margin Algorithm (MMA) to learn the reward function for a robotic navigation problem. The learned reward function reveals the demonstrated policy (expert policy) better than all other poli-

\footnotetext{
${ }^{1}$ Corresponding Author: Antonio Coronato; E-mail: antonio.coronato@icar.cnr.it
} 
cies. Results show that this method has better convergence and learned reward functions through the adopted method represents expert behavior more efficiently.

Shah and De Pietro have surveyed IRL algorithms. The purpose of their paper is to provide an overview and theoretical background of IRL in the field of Machine Learning and Artificial Intelligence.

Aamir et al. have introduced a novel supervised machine learning based approach for breast prediction that embodies Random Forest, Gradient Boosting, Support Vector Machine, Artificial Neural Network and Multilayer Perception methods.

We deeply appreciate the Intelligent Environments 2021 conference organizers for their help on hosting this event.

\section{References}

[1] A. Coronato and A. Cuzzocrea. An innovative risk assessment methodology for medical information systems. IEEE Transactions on Knowledge and Data Engineering, pages 1-1, 2020.

[2] A. Coronato and G. De Pietro. Tools for the rapid prototyping of provably correct ambient intelligence applications. IEEE Transactions on Software Engineering, 38(4):975-991, 2012.

[3] Antonio Coronato, Muddasar Naeem, Giuseppe De Pietro, and Giovanni Paragliola. Reinforcement learning for intelligent healthcare applications: A survey. Artificial Intelligence in Medicine, 109:101964, 2020.

[4] Muddasar Naeem, Giovanni Paragliola, and Antonio Coronato. A reinforcement learning and deep learning based intelligent system for the support of impaired patients in home treatment. Expert Systems with Applications, page 114285, 2020.

[5] Muddasar Naeem, S Tahir H Rizvi, and Antonio Coronato. A gentle introduction to reinforcement learning and its application in different fields. IEEE Access, 2020.

[6] Andrew Y Ng, Stuart J Russell, et al. Algorithms for inverse reinforcement learning. In Icml, volume 1, page 2, 2000.

[7] Martin L Puterman. Markov decision processes: discrete stochastic dynamic programming. John Wiley \& Sons, 2014.

[8] Richard S. Sutton and Andrew G. Barto. Reinforcement Learning: An Introduction. The MIT Press, second edition, 2018. 